\title{
Flow cytometry-based immunophenotypic analysis of primary systemic light chain amyloidosis
}

\author{
XIANGWEN DIAO $^{1}$, JUAN LI $^{1}$, JUAN OUYANG ${ }^{2}$, JUNRU LIU ${ }^{1}$, \\ BEIHUI HUANG ${ }^{1}$, MEILAN CHEN ${ }^{1}$ and JINGLI GU ${ }^{1}$ \\ Departments of ${ }^{1}$ Hematology and ${ }^{2}$ Laboratory Medicine, First Affiliated Hospital, \\ Sun Yat-Sen University, Guangzhou, Guangdong 510080, P.R. China
}

Received April 5, 2016; Accepted December 20, 2016

DOI: $10.3892 / \mathrm{ol} .2017 .5767$

\begin{abstract}
Using flow cytometry, the present study aimed to investigate the immunophenotypic characteristics of malignant plasma cells (PCs) in the bone marrow of patients with primary systemic light chain amyloidosis (AL) compared with the characteristics of patients with multiple myeloma (MM). Flow cytometric results of 51 patients with AL and 150 patients with MM were reviewed. The proportion of total bone marrow PCs in the patients with AL was significantly lower than that in the patients with MM, 1.35\% (0.3-9.5\%) vs. 9.7\% (0.4-75.7\%); $(\mathrm{P}<0.001)$. The cells in $24 / 51$ patients with AL comprised two distinct populations: Normal PCs and malignant PCs, whereas only $11 / 150$ patients with MM exhibited two populations. Patients with AL exhibited an increased cluster of differentiation (CD)19 expression compared with patients with MM, 4.5\% (0.1-80.9\%) vs. $1.6 \%(0.1-33.6 \% ; \mathrm{P}<0.001)$ and reduced CD138, with $90.8 \%$ (30.4-99.9\%) vs. $95.0 \%(40.7-100 \% ; \mathrm{P}=0.006)$ and $\mathrm{CD} 56$, with $61.0 \%(1.5-99.2 \%)$ vs. $98.3 \%(0.1-100 \%$; $\mathrm{P}<0.001)$ expression in malignant PCs. Light chain restriction was identified in all patients with $\mathrm{AL}$, and the proportion of $\lambda$ light chain restriction was $72.5 \%$. The immunophenotypic characteristics of patients with AL demonstrated by flow cytometry were different compared with the characteristics of patients with MM in the number and composition of plasma cells, and the intracellular and extracellular expression of antigens. These differences may be associated with a less malignant phenotype of clonal $\mathrm{PCs}$ in AL than MM.
\end{abstract}

\section{Introduction}

Primary systemic light chain amyloidosis (AL) is a clonal plasma cell (PC) disorder whereby a monoclonal population of

Correspondence to: Dr Juan Li, Department of Hematology, First Affiliated Hospital, Sun Yat-Sen University, 58 2nd Yat-Sen Road, YueXiu, Guangzhou, Guangdong 510080, P.R. China

E-mail: 13719209240@163.com

Key words: primary systemic light chain amyloidosis, multiple myeloma, flow cytometry, immunophenotype bone marrow PCs produces misfolded light chains that leads to multiple organ dysfunction (1). Although the toxic light chain repertoire has been intensively studied in AL, data concerning pathological clonal PCs, particularly their immunophenotypic features remains limited. In 2003, Shimojima et al (2) classified PCs into immature, intermediate and mature stages according to their expression of cluster of differentiation (CD)45, CD49e and MPC-1 antigen (MPC-1), and revealed that the majority of PCs in patients with $\mathrm{AL}$ were of the intermediate subtype (MPC-1+CD45-CD49e-), whilst the majority of the PCs in patients with monoclonal gammopathy of unknown significance (MGUS) were mature (MPC-1+CD45+CD49e-). Paiva et al (3) summarized the immunophenotypes of the malignant PCs in 35 patients with AL: $100 \%$ of the patients were CD38+, $97 \%$ of the patients were CD45dim/-, $91 \%$ of the patients were CD19- and 59\% of the patients were CD56+. The authors proposed that the majority of malignant PC immunophenotypes in patients with AL overlap with the immunophenotypes in patients with multiple myeloma (MM). Previously, a study from China directly compared the immunophenotypic characteristics between 51 patients with AL and 21 patients with MM (4). The authors demonstrated that the two diseases shared similar immunophenotypes, except that the CD117+ rate in patients with AL was higher than that in patients with MM.

Thus far, there have been no well-defined flow cytometry (FCM) immunophenotypic profiles of AL. Therefore, the present study conducted a retrospective analysis of the flow cytometric immunophenotypes in patients with AL and MM who were diagnosed for the first time in the First Affiliated Hospital, Sun Yat-Sen University (Guangzhou, China), to explore the immunological characteristics of amyloidogenic clonal PCs.

\section{Materials and methods}

Subjects. The present study included 51 patients with AL that were diagnosed in the First Affiliated Hospital, Sun Yat-Sen University (Guangzhou, China), between November 2008 and March 2015 as the study group. The baseline clinical characteristics are summarized in Table I. All patients with AL underwent biopsy of the affected tissue, and the presence of amyloidosis was confirmed by Congo red staining and/or 
by electron microscopy. Additional immunohistochemistry with commercially available monoclonal antibodies directed against $\lambda$-light chain [ready-to-use (RTU); cat. no. A0193] and $\kappa$-light chain (RTU; cat. no. IR506) (both Dako; Agilent Technologies, Inc., Santa Clara, CA, USA) suggested that all fibrillar amyloids were composed of immunoglobulin light chains (5). An EnVision ${ }^{\mathrm{TM}}$ FLEX+ Mouse LINKER system (Dako; Agilent Technologies, Inc.) was used to visualize the antigens (cat. no. K800221). A total of 150 newly diagnosed patients with MM were designated as the MM group, and all patients in this group met the 2011 International Myeloma Working Group diagnostic criteria (6). A separate non-plasma cell neoplasm (PCN) control group was also established and was referred to as the control group. This group consisted of 30 patients where the presence of PCN was ruled out by flow cytometric immunophenotypic examination and immunofixation electrophoresis. The 30 patients included 7 patients with primary glomerulonephritis, 4 with acute or chronic renal failure, 5 with respiratory infections, 2 with rheumatoid arthritis, 2 with systemic lupus erythematosus, 2 with Sjogren's syndrome, 1 with aplastic anemia, 1 with lymphoma, 1 with gastric cancer bone metastasis, and 5 with osteoporosis. No statistically significant difference was observed between gender composition $(\mathrm{P}=0.73)$ and age $(\mathrm{P}=0.07)$ among the 3 groups, as demonstrated in Table II. The study was approved by the Investigational Review Board at the First Affiliated Hospital, Sun Yat-Sen University (Guangzhou, China), and written informed consent was obtained from all patients involved.

Morphological examination of bone marrow cells. Morphological evaluation was performed independently without knowledge of the flow cytometry findings. Each case was reviewed at least by one hematopathologist, and the diagnosis of cases that fulfilled the initial inclusion criteria was confirmed by another hematopathologist for this study. For each case, Wright-Giemsa stained BM aspirate smears were evaluated. A cell count of 500 was performed based on examination of multiple fields of BM aspirate smears via light microscopy. Cases with inadequate quality of BM smears were not included in this study.

Specimen collection, preparation, immunofluorescence labeling, and flow cytometry analysis. BM aspirate samples were collected in EDTA-anticoagulant and processed within $24 \mathrm{~h}$ of collection. Following incubation with monoclonal antibodies for $20 \mathrm{~min}$ at $20^{\circ} \mathrm{C}$, erythrocytes were lysed with ammonium chloride (PharmLyse ${ }^{\mathrm{TM}}$; BD Biosciences, San Jose, CA, USA) at room temperature for $10 \mathrm{~min}$ using a standard lyse/wash technique (7). The antibody information is illustrated in Table III. Samples were acquired on fluorescence FACSCanto instrument (BD Biosciences). A minimum of 100,000 events were acquired. In certain cases with a low number of PC cells, $\leq 500,000$ events were acquired to obtain $\geq 100$ cells in the PC gate in all tubes. Data were analyzed using FACSDiva software (version 8.0.1; BD Biosciences). Analysis of the surface and cytoplasmic markers of the plasma cells was performed using a 6-color panel of RTU antibodies and isotype controls conjugated to fluorescein isothiocyanate (FITC), phycoerythrin (PE), peridinin chlorophyll, PE-cyanine 7 (PE-Cy7), allophycocyanin (APC) and/or
Table I. Baseline characteristics of patients with light-chain amyloidosis.

\begin{tabular}{lc}
\hline Characteristic & $\begin{array}{c}\text { Value [median } \\
\text { (min-max) or } \\
\text { no. }(\%)]\end{array}$ \\
\hline Serum albumin, g/dl & $2.7(1.4-4.2)$ \\
Serum creatinine, $\mu$ mol/1 & $144.42(33-713)$ \\
Serum alkaline phosphatase, IU/1 & $107.14(36-552)$ \\
ß2-Microglobulin, mg/l & $5.4(1.1-38.5)$ \\
Urine total protein, g/24 h & $4.74(0.05-16.62)$ \\
Serum M-component, g/l & $6.8(0-23.5)$ \\
Urine M-component, g/24 h & $0.14(0-3.37)$ \\
Serum cardiac troponin T, ng/ml & $0.26(0.0-5.03)$ \\
NT-ProBNP, pg/ml & $10,304(6-76,874)$ \\
Number of organs involved & \\
2 & $27 / 51(52.9)$ \\
$\geq 3$ & $24 / 51(47.1)$ \\
Kidney involvement & $41 / 51(80.4)$ \\
Heart involvement & $32 / 44(72.7)$ \\
Liver involvement & $8 / 47(17.0)$ \\
Peripheral nerve involvement & $21 / 25(84.0)$ \\
\hline
\end{tabular}

NT-ProBNP, N-terminal pro B-type natriuretic peptide.

APC-Cy7. The antibody panel included: i) Anti-CD38, -CD56, -CD19, -CD20, -CD138 and -CD45; ii) anti-cytoplasmic (c)к, - (c) $\lambda$, -CD56, -CD138, -CD38 and -CD45; and iii) anti-CD38, -CD54, -CD138 and -CD45 (all RTU; Table III).

CD38/CD138/CD45/side scatter (SSC) was used as the gate to identify the subpopulations of PCs in the bone marrow. The levels of expression of CD19 and CD56 on the cell surface and the cytoplasmic expression of light chains was analyzed to distinguish between benign and malignant PCs $(8,9)$. Cell surface expression levels $>20 \%$ for CD19, CD20, CD56, CD54 or CD138 were defined as positive.

Statistical analysis. Unless noted specifically, all data in the present study are expressed as the median (range). Normally distributed data were compared using unpaired Student's t-test or analysis of variance. Data that were not normally distributed were tested using the Mann-Whitney $\mathrm{U}$ test and ratios were compared using the $\chi^{2}$ test. $\mathrm{P}<0.05$ was considered to indicate a statistically significant difference. Receiver operating characteristic (ROC) curves were created to identify cut-off values for the flow cytometry indexes to differentiate patients with AL and MM. Data collection, management and statistical analysis were conducted using SPSS (version 17.0; SPSS, Inc., Chicago, IL, USA).

\section{Results}

Patients with AL exhibited lower bone marrow PC proportions compared with patients with MM. Whether measured 
Table II. Patient demographics.

\begin{tabular}{lcrr}
\hline Variable & AL group & MM group & Control group \\
\hline Male $(\%)$ & $32 / 50(62.7)$ & $100 / 150(66.7)$ & $18 / 30(60.0)$ \\
Age [Median (min-max)] & $58(33-79)$ & $56(31-75)$ & $52(27-77)$ \\
\hline
\end{tabular}

AL, primary systemic light chain amyloidosis; MM, multiple myeloma.

Table III. Antibodies used for flow cytometry.

\begin{tabular}{|c|c|c|c|}
\hline Antibodies & Cat. no. & $\begin{array}{l}\text { Concentration } \\
(\mu 1 / 100 \mu 1)\end{array}$ & Manufacturer \\
\hline $\begin{array}{l}\text { Anti-CD38-FITC/CD56-PE/ } \\
\text { CD19-PerCP-Cy5.5 }\end{array}$ & 341132 & 20 & $\begin{array}{l}\text { BD Biosciences, San Jose, } \\
\text { CA, USA }\end{array}$ \\
\hline Anti-CD20-PE-Cy7 & 335793 & 5 & BD Biosciences \\
\hline Anti-CD38 (HB-7)-APC & 345807 & 5 & BD Biosciences \\
\hline Anti-CD54-PE & 555511 & 20 & BD Biosciences \\
\hline Anti-CD138-PerCP-Cy5.5 & 341087 & 20 & BD Biosciences \\
\hline 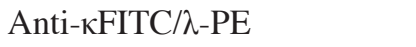 & 349516 & 20 & BD Biosciences \\
\hline Anti-CD45-APC & 340943 & 5 & BD Biosciences \\
\hline
\end{tabular}

$\mathrm{CD}$, cluster of differentiation; FITC, fluorescein isothiocyanate; PE, phycoerythrin; PerCP, peridinin chlorophyll; Cy, cyanine; APC, allophycocyanin.

by cell morphology or FCM, the majority of patients with AL exhibited bone marrow PC proportions of $<10 \%$, as demonstrated in Table IV. Patients with AL exhibited a lower total PC proportion by FCM compared with patients with MM, $1.35 \%(0.3-9.5 \%)$ vs. $9.7 \%(0.4-75.7 \%$; $\mathrm{P}<0.001)$. The optimal cut-off value of the total PC proportion by FCM to distinguish between the two was $4.2 \%$ (area under the ROC curve $=0.88 ; \mathrm{P}<0.001)$. The sensitivity was $92.2 \%$ and the specificity was $72.7 \%$.

A total of two populations of malignant and normal PCs were detected more frequently in AL compared with MM. Amongst the 51 patients with AL, benign and malignant PCs were detected in the bone marrow samples from 24 patients, whereas the two populations of PCs were detected in only $11 / 150$ patients with MM, and the difference was statistically significant (47.1 vs. 7.3\%; $\mathrm{P}<0.001$ ), as demonstrated in Fig. 1. The 11 patients with MM exhibiting two populations of PCs demonstrated higher malignant PC proportions among the total PCs compared with the 24 patients with AL who exhibited two populations of PCs, $90.67 \%$ (50.0-98.36\%) vs. $76.39 \%$ (33.33-91.67\%; $\mathrm{P}=0.008)$, as illustrated in Fig. 2.

Malignant PCs were slightly smaller in patients with AL than in patients with MM. The size and granularity of the PCs were defined by forward scatter and SSC, respectively. Malignant PCs were smaller in patients with AL compared with patients with MM ( $\mathrm{P}=0.009)$, as demonstrated in Table V and Fig. 3 , although the difference in cell granularity was not statistically significant $(\mathrm{P}=0.473)$. AL malignant $\mathrm{PCs}$ were larger
$(\mathrm{P}=0.021)$ but showed less granularity $(\mathrm{P}=0.014)$ compared with the control PC group.

Patients with AL and MM differed in malignant PC surface antigen expression. The expression patterns of the surface antigens in malignant bone marrow PCs from patients with $\mathrm{AL}$ and $\mathrm{MM}$ and those in the PCs from the control group are illustrated in Table VI. Bright expression of CD38 was detected in all patients. CD19 expression on the surface of malignant PCs increased in patients with AL, while CD138 and CD56 expression levels decreased compared with patients with MM.

CD138 expression levels on the surface of malignant PCs were lower in AL patients than in MM patients. The CD138 expression level was lower in patients with AL compared with patients with MM, 90.8\% (30.4-99.9\%) vs. 95.0\% (40.7-100\%; $\mathrm{P}=0.006)$, but was higher compared with the control group, $81.0 \%$ (29.7-95.2\%; $\mathrm{P}=0.024)$. The ROC curve showed that the best critical point of CD138 expression to differentiate between AL and MM was $94.25 \%$ (area under the ROC curve $=0.63$; $\mathrm{P}=0.003$ ), with a sensitivity of $72.5 \%$ and a specificity of $53.3 \%$.

CD19 expression levels on the surface of malignant PCs were higher in patients with AL compared with patients with MM. Of the 51 patients with AL, 7 exhibited positive CD19 expression whereas only 4/150 patients with MM were CD19+, a difference that was statistically significant, 13.7 vs. $2.7 \%$ $(\mathrm{P}=0.007)$. The $\mathrm{CD} 19$ expression level was higher in patients with AL compared with patients with MM, 4.5\% (0.1-80.9\%) 
Table IV. Bone marrow plasma cell counts assessed by morphology and FCM.

\begin{tabular}{|c|c|c|c|c|}
\hline Variable & AL group & MM group & Control group & P-value \\
\hline PCs by morphology (\%) & $4(0.5-16)^{\mathrm{a}, \mathrm{b}}$ & $24(3-85)$ & $1.5(0.5-12)$ & $<0.01$ \\
\hline PCs by FCM $(\%)$ & $1.35(0.3-9.5)^{\mathrm{a}, \mathrm{b}}$ & $9.7(0.4-75.7)^{\mathrm{a}}$ & $0.7(0.1-4.6)^{\mathrm{b}}$ & $<0.01$ \\
\hline M-PCs by FCM (\%) & $1.10(0.14-9.5)^{\mathrm{a}, \mathrm{b}}$ & $9.7(0.24-75.7)^{\mathrm{a}}$ & & $<0.01$ \\
\hline
\end{tabular}

${ }^{\mathrm{a}} \mathrm{P}<0.05$ vs. control group. ${ }^{\mathrm{b}} \mathrm{P}<0.001$ vs. MM group. Data are expressed as the median (range). FCM, flow cytometry; PCs, plasma cells; M-PCs, monoclonal PCs; AL, primary systemic light chain amyloidosis; MM, multiple myeloma.
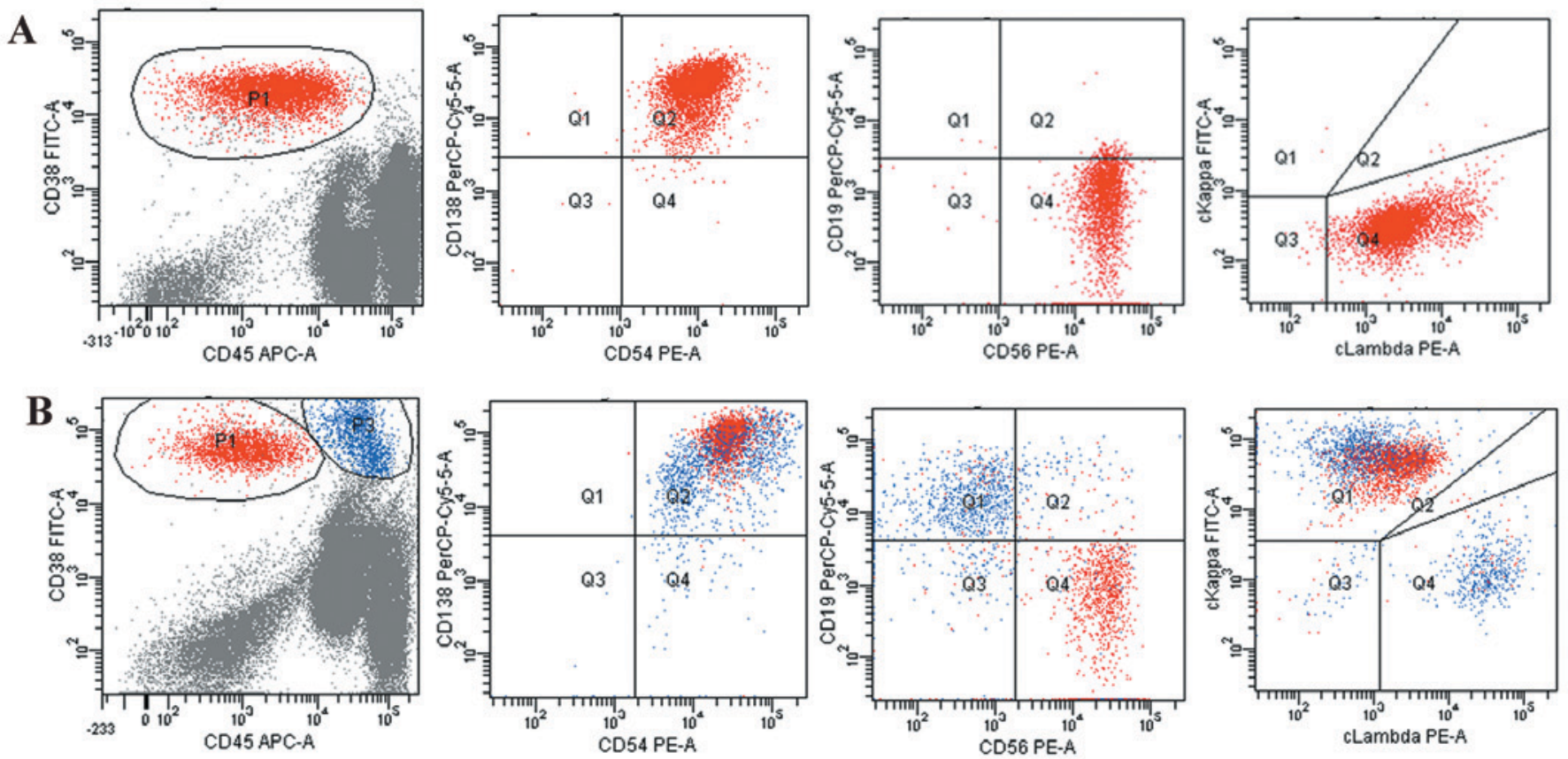

Figure 1. Examples of typical immunophenotypes and clonality assessments of PCs in bone marrow samples from the AL and MM groups. (A) Immunophenotypes of malignant bone marrow PCs in one patient with MM with the immunophenotype CD38+CD45-/dimCD138+CD19-CD56+ and an abnormal cytoplasmic $\kappa / \lambda$ light chain ratio. (B) Flow cytometry results from one patient with AL. Malignant PC populations without normal PCs were detected in the majority of bone marrow samples from patients with MM (139/150; 92.7\%). Whereas two distinct populations, normal PCs and malignant PCs, were detected in $\sim 50 \%$ of patients with AL $(24 / 51 ; 47.1 \%)$. CD, cluster of differentiation; APC-A, allophycocyanin-A; PE-A, phycoerythrin-A; FITC-A, fluorescein isothiocyanate; AL, primary systemic light chain amyloidosis; MM, multiple myeloma; PC, clonal plasma cell; P1, malignant plasma cells, with the immunophenotype CD38+CD45-/dimCD138+CD19-CD56+ and an abnormal cytoplasmic $\kappa / \lambda$ light chain ratio; P3, normal plasma cells with the immunophenotype CD38+CD45+CD138+CD19+CD56- and a cytoplasmic $\kappa / \lambda$ ratio of 1.98 .

vs. $1.6 \%(0.1-33.6 \%$; $\mathrm{P}<0.001)$. The majority of the control group patients, $93.3 \%$, exhibited CD19 expression on their PC surfaces and demonstrated high expression levels, $71.8 \%$ (0.4-90.3\%). The ROC curve showed that the best critical point of CD19 expression for the distinction between the AL and $\mathrm{MM}$ groups was $2.5 \%$ (area under the ROC curve $=0.74$; $\mathrm{P}<0.001$ ), with a sensitivity of $72.5 \%$ and a specificity of $67.3 \%$.

The CD56 expression levels on the surface of malignant PCs were lower in patients with AL compared with patients with $M M$. The CD56+ expression rate on the surface of malignant PCs was lower in patients with AL, 58.8\%, compared with patients with MM, 76.7\% ( $\mathrm{P}=0.014)$. In addition, the CD56 expression level was significantly lower in patients with $\mathrm{AL}$, $61.0 \%(1.5-99.2 \%)$ compared with patients with MM, 98.3\% (0.1-100\%; $\mathrm{P}<0.001)$. The ROC curve demonstrated that the best critical point of CD56 expression to differentiate between the two groups was $95.8 \%$ (area under the ROC curve $=0.768$; $\mathrm{P}<0.001$ ), with a sensitivity of $94.1 \%$ and a specificity of $59.3 \%$.

Ratio of patients with predominant $\lambda$ light chain in the cytoplasm of malignant PCs was higher in the AL group compared with the MM group. The cytoplasmic $\kappa$ and $\lambda$ light chain ratio was abnormal in the malignant bone marrow PCs from patients with AL and MM. The proportion of $\lambda$ light chain restriction in patients with AL was $72.5 \%$, which was higher compared with the proportion from patients with MM, 72.5 vs. $36.7 \%$ $(\mathrm{P}<0.001)$.

\section{Discussion}

Although AL and MM exhibit different biological behaviors and clinical phenotypes, it has been demonstrated that the 
Table V. Comparison of the granularity and size of clonal PCs from AL and MM patients and normal bone marrow PCs from the control group.

\begin{tabular}{lccc}
\hline Variable & AL group & MM group & Control group \\
\hline FSC & $10,7675(29,778-190,691)^{\mathrm{a}, \mathrm{b}}$ & $129,675(62,814-216,102)$ & $100,577(20,944-185,665)^{\mathrm{a}}$ \\
SSC & $1,664(464-5,259)^{\mathrm{b}}$ & $1,503(287-4,751)^{\mathrm{b}}$ & $2,052(533-3,790)$ \\
\hline
\end{tabular}

${ }^{\mathrm{a}} \mathrm{P}<0.01$ vs. MM group. ${ }^{\mathrm{b}} \mathrm{P}<0.05$ vs. control group. Data are expressed as the median (range). AL, primary systemic light chain amyloidosis; MM, multiple myeloma; PCs, plasma cells; FSC, forward scatter; SSC, side scatter.

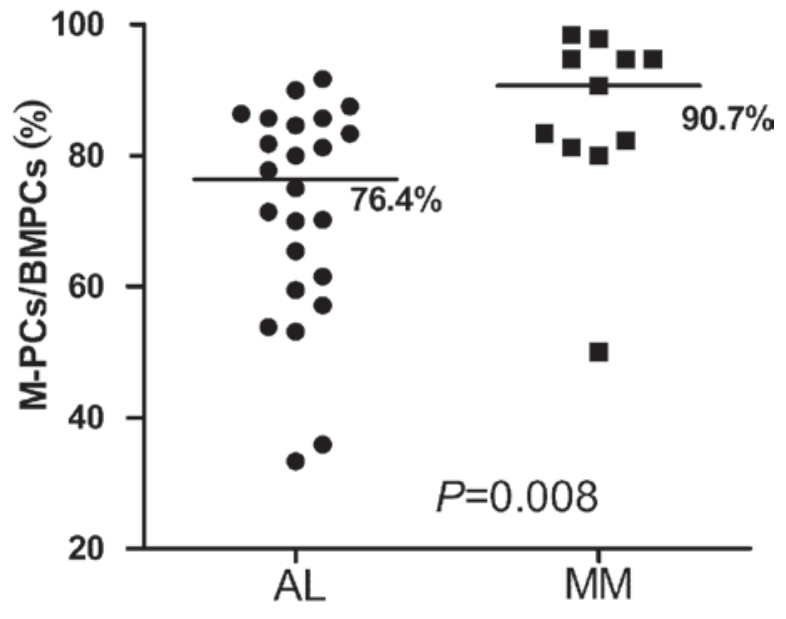

Figure 2. Different ratios of M-PCs/BMPCs between the AL and MM groups. Patients with MM who had two populations of PCs exhibited higher M-PCs proportions among the total PCs compared with patients with AL who had two populations of PCs [90.67\% (50.0-98.36\%) vs. 76.39\% (33.33-91.67\%); $\mathrm{P}=0.008]$. PCs, plasma cells; M-PCs, monoclonal plasma cells; BMPCs, bone marrow plasma cells; AL, primary systemic light chain amyloidosis; MM, multiple myeloma.

FCM immunophenotypes of patients with AL overlap with the immunophenotypes of patients with MM (10). AL is hypothesized to exhibit the same disease pattern as MGUS and $\mathrm{MM}$ at the cellular level, with AL being a clonal PC disorder with an 'unlucky protein' (11). In the present study, the differences between AL and MM immunophenotypes were systematically compared by FCM. The results indicated that in patients with $\mathrm{AL}$, the proportion of total bone marrow PCs was significantly lower compared with patients with $\mathrm{MM}$ and 2 distinct populations, benign cells and malignant cells, were detected in almost half of patients. In addition, the expression of CD19 was higher and the expression of CD56 and CD138 was lower in patients with AL compared with patients with MM.

Consistent with a previous study, the average PC proportion among all bone marrow nucleated cells in the present study was lower in patients with AL compared with patients with MM (12). The proportion of PCs measured by FCM was lower compared with the proportion of PCs measured by the bone marrow smear examinations as; FCM includes bone marrow aspiration, whereby bone grease may be washed away during the specimen preparation, and certain PCs may adhere to the bone grease and the bone marrow aspirate may mix with peripheral blood in the extraction process (13).
In addition to the $\mathrm{PC}$ proportion, patients with $\mathrm{AL}$ and $\mathrm{MM}$ also differed significantly with respect to the composition of PCs. Almost $50 \%$ of the bone marrow samples from patients with AL exhibited two types of PC with normal and abnormal immunophenotypes, as detected by flow cytometry. However, PCs exhibiting a normal immunophenotype were only detected in $7.3 \%$ of patients with MM. When comparing patients with AL and MM with two populations of PCs, the ratio of malignant PCs in patients with AL was significantly lower compared with patients with MM. In addition to AL, the populations of benign and malignant cells may also be detected in almost all of the patients with MGUS, a premalignant condition of MM (14). Furthermore, the proportion of normal PCs amongst the total PCs may assist to distinguish MGUS from MM, therefore screening patients with a high risk of progressing to MM $(14,15)$. It may be suggested that the proliferation index and invasiveness of malignant PCs in AL were more similar to those in MGUS compared with MM.

CD19 is a B cell-specific marker and exhibits almost no expression on the surface of malignant PCs (16). The absence of CD19 in MM PCs suggests the deteriorating progression of tumor cells (17). Mahmoud et al (17) increased the cell surface expression levels of CD19 in myeloma cell lines and revealed that tumor cell growth was inhibited and the capacity to generate tumor cells was reduced. The results of the present study indicate that in patients with AL, the CD19 expression in the malignant PCs was significantly higher compared with patients with MM, which may also suggest that AL is less malignant than MM.

CD138, also termed syndecan-1, is one of the specific markers of normal PCs and malignant PCs. CD138 binds to soluble molecules such as epidermal growth factor, fibroblast growth factor and hepatocyte growth factor, and insoluble molecules such as collagen and fibronectin via the terminal heparin sulfate chains. Thus, CD138 mediates cell-cell adhesion and the interaction between cells, and promotes the proliferation and growth of malignant PCs (18). In addition, CD138 is also a PC differentiation antigen. CD138- plasma blasts are $\mathrm{PC}$ progenitor cells, which differentiate into CD138+ PC precursors and finally mature into $\mathrm{CD} 138++$ PCs that no longer possess the capacity for mitosis and proliferation (19). The present study demonstrated that the CD138 expression level on the surface of malignant PCs in patients with AL was lower compared with patients with MM, suggesting that clonal PCs from patients with AL may differ from the PCs of patients with MM in terms of proliferation, growth and interaction with the bone marrow microenvironment. 
Table VI. Immunophenotypic characteristics of clonal PCs from the AL and MM groups and bone marrow plasma cells from the control group presented as proportions of cell subpopulations expressing the analyzed antigen.

\begin{tabular}{lcccccc}
\hline & & & & \multicolumn{3}{c}{ P-value } \\
\cline { 5 - 7 } & AL group & MM group & Control group & $\begin{array}{c}\text { AL vs. } \\
\text { MM }\end{array}$ & $\begin{array}{c}\text { AL vs. } \\
\text { control }\end{array}$ & $\begin{array}{c}\text { MM vs. } \\
\text { control }\end{array}$ \\
\hline CD138 & $90.8 \%(30.4-99.9 \%)$ & $95.0 \%(40.7-100 \%)$ & $81.0 \%(29.7-95.2 \%)$ & 0.006 & 0.024 & $<0.001$ \\
CD19 & $4.5 \%(0.1-80.9 \%)$ & $1.6 \%(0.1-33.6 \%)$ & $71.8 \%(0.4-90.3 \%)$ & $<0.001$ & $<0.001$ & $<0.001$ \\
CD20 & $4.7 \%(0.1-95.5 \%)$ & $4.3 \%(0.1-99.6 \%)$ & $5.8 \%(1.0-22.0 \%)$ & 0.40 & 0.37 & 0.067 \\
CD54 & $99.1 \%(61.2-100 \%)$ & $98.6 \%(27.2-100 \%)$ & $97.9 \%(70.8-99.9 \%)$ & 0.13 & 0.07 & 0.59 \\
CD56 & $61.0 \%(1.5-99.2 \%)$ & $98.3 \%(0.1-100 \%)$ & $7.1 \%(0.7-41.7 \%)$ & $<0.001$ & 0.003 & $<0.001$ \\
\hline
\end{tabular}

All cases were CD38+. Data are expressed as the median (range). AL, primary systemic light chain amyloidosis; MM, multiple myeloma; CD, cluster of differentiation.

Table VII. Comparison of our data with major studies that focused on the malignant plasma cell phenotypic profile in AL.

\begin{tabular}{lcccc}
\hline & Present study & Hu et al (4) & Paiva et al (3) & Yoshida et al (22) \\
\hline Year published & & 2014 & 2011 & 2008 \\
Number of patients & 51 & 51 & 35 & 59 \\
M-PCs by FCM (\%) & $1.10(0.14-9.5)^{\mathrm{a}}$ & $3.87(0.17-9.34)^{\mathrm{a}}$ & $1.4(0.1-15.0)^{\mathrm{a}}$ & $2.16 \pm 2.12^{\mathrm{b}}$ \\
CD45-/dim (\%) & 98 & 100 & 97 & - \\
CD56+ (\%) & 58.8 & 78.4 & 59 & - \\
CD19+ (\%) & 13.7 & 11.8 & 9 & - \\
CD20+ (\%) & 11.8 & 13.7 & - & - \\
Others (\%) & LCR 100 & LCR 60.8, & - & - \\
& & CD117+69 & & \\
\hline
\end{tabular}

${ }^{a}$ Data are expressed as the median (range); bdata are expressed as the mean \pm standard deviation; LCR, light chain restriction; M-PCs, monoclonal plasma cells; FCM, flow cytometry; CD, cluster of differentiation.
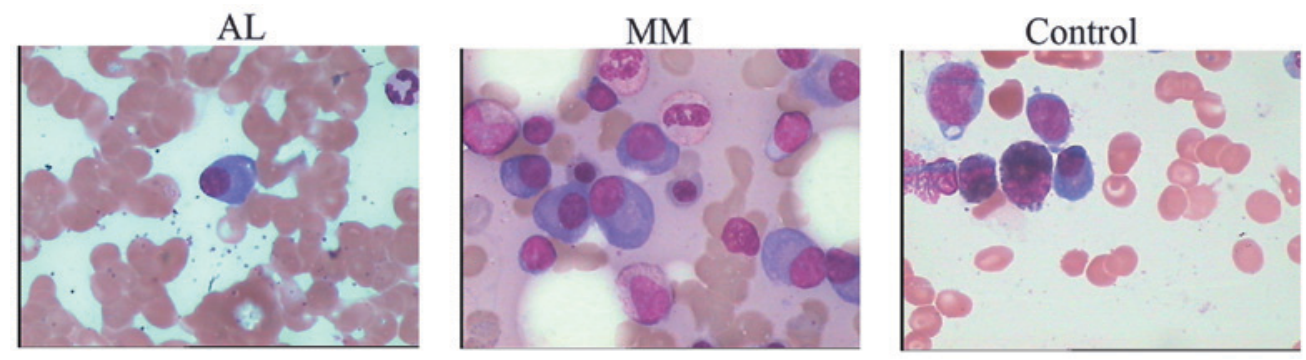

Figure 3. Morphological differences in abnormal plasma cell clones between the AL and MM groups and bone marrow plasma cells from the control group (Wright-Giemsa staining; magnification, x1,000). AL, primary systemic light chain amyloidosis; MM, multiple myeloma.

Moreover, AL may also exhibit lower PC differentiation degrees compared with MM.

The present study also revealed that the level of CD56 expression significantly increased in MM malignant clonal PCs and was relatively low in patients with AL. Ely and Knowles (20) reported that CD56 expression in PCs is associated with osteolytic lesions in patients with myeloma. The CD56 molecule facilitates homeopathic interactions between myeloma cells and osteoblasts, resulting in a decreased generation of bone matrix and eventually osteolytic bone changes (21). The data from the present study suggest that the interaction between malignant bone marrow PCs and the bone matrix in patients with $\mathrm{AL}$ is not as strong as the interaction in $\mathrm{MM}$ tumor cells. The data suggest that the two diseases differ in the bone marrow microenvironment surrounding the malignant bone marrow PCs, and that there may be different signaling pathways and interactions affecting the biological behavior of clonal PCs in each disease. 
The present study and previous major studies on AL immunophenotypes are summarized in Table VII. The advantage of the present study is that the benign and malignant PCs in the bone marrow from each patient were first distinguished, then the immunophenotypes of the malignant PCs were analyzed. This is particularly important in the patients with AL as the invasiveness of malignant PCs and the ability of malignant PCs to reproduce in patients with AL are not as strong, and the suppression of the normal PCs is relatively weak. A possible reason for the high proportion of PCs in AL obtained by $\mathrm{Hu}$ et al (4) is that the benign and malignant bone marrow PCs were not distinguished. In addition, unlike the studies of Paiva et al (3) and Yoshida et al (22), the present study collected the flow cytometry data for patients with AL and $\mathrm{MM}$, and demonstrated the immunophenotypic characteristics of AL malignant PCs clearly with a systematic comparison. The main disadvantages of the present study are that it is a retrospective analysis and the antigens for analysis are limited.

In conclusion, the flow cytometric immunophenotypic characteristics of AL are different compared to the characteristics of MM in the number and the composition of PCs, and the intracellular and extracellular expression of antigens, which may be associated with a less malignant phenotype of clonal PCs in AL compared with MM.

\section{Acknowledgements}

The present study was supported by the Program of National Key Clinical Specialties and Science and Technology Planning Project of Guangdong Province, China (grant no. 2014A020212061).

\section{References}

1. Merlini G, Wechalekar AD and Palladini G: Systemic light chain amyloidosis: An update for treating physicians. Blood 121: 5124-5130, 2013

2. Shimojima Y, Matsuda M, Gono T, Ishii W, Fushimi T, Hoshii Y, Yamada $\mathrm{T}$ and Ikeda S: Correlation between serum levels of free light chain and phenotype of plasma cells in bone marrow in primary AL amyloidosis. Amyloid 12: 33-40, 2005.

3. Paiva B, Vidriales MB, Pérez JJ, López-Berges MC, García-Sanz R, Ocio EM, de Las Heras N, Cuello R, García de Coca A, Pardal E, et al: The clinical utility and prognostic value of multiparameter flow cytometry immunophenotyping in light-chain amyloidosis. Blood 117: 3613-3616, 2011.

4. Hu Y, Wang M, Chen Y, Chen X, Fang F, Liu S, Zhang Y, Wu X and Zhu P: Immunophenotypic analysis of abnormal plasma cell clones in bone marrow of primary systemic light chain amyloidosis patients. Chin Med J (Engl) 127: 2765-2770, 2014.

5. Gertz MA, Comenzo R, Falk RH, Fermand JP, Hazenberg BP, Hawkins PN, Merlini G, Moreau P, Ronco P, Sanchorawala V, et al: Definition of organ involvement and treatment response in immunoglobulin light chain amyloidosis (AL): A consensus opinion from the 10th international symposium on amyloid and amyloidosis, Tours, France, 18-22 April 2004. Am J Hematol 79: 319-328, 2005.

6. Dimopoulos M, Kyle R, Fermand JP, Rajkumar SV, San Miguel J, Chanan-Khan A, Ludwig H, Joshua D, Mehta J, Gertz M, et al: Consensus recommendations for standard investigative workup: Report of the international myeloma workshop consensus panel 3. Blood 117: 4701-4705, 2011.

7. Cannizzo E, Bellio E, Sohani AR, Hasserjian RP, Ferry JA, Dorn ME, Sadowski C, Bucci JJ, Carulli G and Preffer F: Multiparameter immunophenotyping by flow cytometry in multiple myeloma: The diagnostic utility of defining ranges of normal antigenic expression in comparison to histology. Cytometry B Clin Cytom 78: 231-238, 2010.
8. San MJ, Almeida J, Mateo G, Bladé J, López-Berges C, Caballero D, Hernández J, Moro MJ, Fernández-Calvo J, Díaz-Mediavilla J, et al: Immunophenotypic evaluation of the plasma cell compartment in multiple myeloma: A tool for comparing the efficacy of different treatment strategies and predicting outcome. Blood 99: 1853-1856, 2002.

9. Morice WG, Hanson CA, Kumar S, Frederick LA, Lesnick CE and Greipp PR: Novel multi-parameter flow cytometry sensitively detects phenotypically distinct plasma cell subsets in plasma cell proliferative disorders. Leukemia 21: 2043-2046, 2007.

10. Filipova J, Rihova L, Vsianska P, Kufova Z, Kryukova E, Kryukov F and Hajek R: Flow cytometry in immunoglobulin light chain amyloidosis: Short review. Leuk Res: Jul 13, 2015 (Epub ahead of print).

11. Bochtler T, Hegenbart U, Cremer FW, Heiss C, Benner A, Hose D, Moos M, Bila J, Bartram CR, Ho AD, et al: Evaluation of the cytogenetic aberration pattern in amyloid light chain amyloidosis as compared with monoclonal gammopathy of undetermined significance reveals common pathways of karyotypic instability. Blood 111: 4700-4705, 2008.

12. Kyle RA and Gertz MA: Primary systemic amyloidosis: Clinical and laboratory features in 474 cases. Semin Hematol 32: 45-59, 1995.

13. Paiva B, Almeida J, Pérez-Andrés M, Mateo G, López A, Rasillo A, Vidriales MB, Lopez-Berges MC, Miguel JF and Orfao A: Utility of flow cytometry immunophenotyping in multiple myeloma and other clonal plasma cell-related disorders. Cytometry B Clin Cytom 78: 239-252, 2010.

14. Ocqueteau M, Orfao A, Almeida J, Bladé J, González M, García-Sanz R, López-Berges C, Moro MJ, Hernández J, Escribano L, et al: Immunophenotypic characterization of plasma cells from monoclonal gammopathy of undetermined significance patients. Implications for the differential diagnosis between MGUS and multiple myeloma. Am J Pathol 152: 1655-1665, 1998.

15. Perez-Persona E, Vidriales MB, Mateo G, García-Sanz R, Mateos MV, de Coca AG, Galende J, Martín-Nuñez G, Alonso JM, de Las Heras N, et al: New criteria to identify risk of progression in monoclonal gammopathy of uncertain significance and smoldering multiple myeloma based on multiparameter flow cytometry analysis of bone marrow plasma cells. Blood 110: 2586-2592, 2007.

16. Bataille R, Jégo G, Robillard N, Barillé-Nion S, Harousseau JL, Moreau P, Amiot M and Pellat-Deceunynck C: The phenotype of normal, reactive and malignant plasma cells. Identification of 'many and multiple myelomas' and of new targets for myeloma therapy. Haematologica 91: 1234-1240, 2006.

17. Mahmoud MS, Fujii R, Ishikawa $\mathrm{H}$ and Kawano MM: Enforced CD19 expression leads to growth inhibition and reduced tumorigenicity. Blood 94: 3551-3558, 1999.

18. Reijmers RM, Spaargaren M and Pals ST: Heparan sulfate proteoglycans in the control of B cell development and the pathogenesis of multiple myeloma. FEBS J 280: 2180-2193, 2013.

19. Jego G, Robillard N, Puthier D, Amiot M, Accard F, Pineau D, Harousseau JL, Bataille R and Pellat-Deceunynck C: Reactive plasmacytoses are expansions of plasmablasts retaining the capacity to differentiate into plasma cells. Blood 94: 701-712, 1999.

20. Ely SA and Knowles DM: Expression of CD56/neural cell adhesion molecule correlates with the presence of lytic bone lesions in multiple myeloma and distinguishes myeloma from monoclonal gammopathy of undetermined significance and lymphomas with plasmacytoid differentiation. Am J Pathol 160: 1293-1299, 2002.

21. Sahara N, Takeshita A, Shigeno K, Fujisawa S, Takeshita K, Naito K, Ihara M, Ono T, Tamashima S, Nara K, et al: Clinicopathological and prognostic characteristics of CD56-negative multiple myeloma. Br J Haematol 117: 882-885, 2002.

22. Yoshida T, Matsuda M, Katoh N, Tazawa K, Shimojima Y, Gono T, Ishii W, Nakazawa Y, Sakashita K, Koike K, et al: Long-term follow-up of plasma cells in bone marrow and serum free light chains in primary systemic AL amyloidosis. Intern Med 47: 1783-1790, 2008. 